\section{Asma não controlada em crianças e adolescentes expostos aos agrotóxicos em região de intensa atividade do agronegócio}

\author{
Uncontrolled asthma in children and adolescents \\ exposed to pesticides in an area of intense \\ agribusiness activity
}

\section{Asma no controlado en niños y adolescentes expuestos a pesticidas en una región de intensa actividad agroindustrial}

Cyndielle Barcelos da Rocha 1 Alessandra Pinheiro Costa Nascimento 1 Ageo Mário Cândido da Silva 1 Clóvis Botelho 1

doi: 10.1590/0102-311X00072220

\section{Resumo}

O objetivo deste estudo é analisar os fatores associados à asma não controlada em escolares expostos aos agrotóxicos em município de médio porte de Mato Grosso, Brasil. Estudo do tipo caso controle, realizado com escolares de 6 a 7 anos e 13 a 14 anos de Primavera do Leste, em 2016. Foram considerados casos, escolares que preencheram critérios para asma não controlada por meio de questões do International Study of Asthma and Allergies in Childhood (ISAAC), os controles foram selecionados a partir das mesmas escolas dos casos, após randomização, numa relação de 1:1. Para a coleta de dados, foram utilizados os questionários da Fase I e II do ISAAC e o questionário adicional para a exposição aos agrotóxicos. Realizaram-se a análise descritiva, bivariada e regressão logística das variáveis sociodemográficas e econômicas, individuais e ambientais. Foram selecionados 319 casos e 319 controles, totalizando em 638 participantes do estudo. No modelo final da regressão logística, as variáveis renda familiar maior que quatro salários mínimos $(O R=14,36$; IC95\%: 8,89-23,20), ter mãe com escolaridade até Ensino Médio incompleto $(O R=16,32$; IC95\%: 8,96-29,75), prematuridade (OR = 13,25; IC95\%: 4,83$36,41)$ e baixo peso ao nascer $(O R=17,08$; IC95\%: 5,52-52,90) mantiveram-se associadas à asma não controlada. Das variáveis de exposição aos agrotóxicos, presença de pessoas no domicílio que trabalham na agricultura $(O R=5,91$; IC95\%: 2,11-16,53), residir próximo da atividade agrícola $(O R=3,98$; IC95\%: 1,47-11,76) e a pulverização aérea próxima ao domicílio $(O R=4,20$; IC95\%: 1,49-11,87) relacionaram-se ao desfecho. Neste estudo, os agrotóxicos $e$ as condições sociodemográficas e de nascimento e infância mostraram-se relacionados à asma não controlada em escolares.

Asma; Agrotóxicos; Fatores de Risco

\author{
Correspondência \\ C. B. Rocha \\ Rua Veu de Noiva 288, Cuiabá, MT 78048-264, Brasil. \\ cyndielle_barcelos@hotmail.com
}

1 Universidade Federal de Mato Grosso, Cuiabá, Brasil. 


\section{Introdução}

De acordo com a classificação da Iniciativa Global para a Asma (GINA), a asma pode ser controlada, parcialmente controlada ou sem controle. A asma não controlada é caracterizada pela presença de sintomas frequentes diurnos e/ou noturnos, limitação das atividades diárias, utilização de medicações de resgate e exacerbações 1 .

Estudo que avaliou o controle da asma em adultos e crianças de países latino-americanos, incluindo o Brasil, evidenciou que somente 2,3\% dos adultos e 2,6\% das crianças preenchiam os critérios de controle total da doença, sendo que mais da metade dos entrevistados visitaram os serviços de emergência devido a sintomas da asma no ano da entrevista 2 . Estudos internacionais mostram que cerca de $50 \%$ dos asmáticos possuem asma não controlada 3,4,5,6, revelando que, apesar da existência de uma estratégia global para o manejo da asma e da disponibilidade de diretrizes nacionais e tratamento eficazes, as taxas de baixo controle da doença permanecem elevadas em todo o mundo 7 .

$\mathrm{O}$ controle inadequado da asma reduz a qualidade de vida e a produtividade dos pacientes e familiares, aumentado o absenteísmo escolar e laboral 8,9. O ônus da doença possui ainda aspectos emocionais, levando à ansiedade, à depressão e ao medo, o que pode comprometer a vida social 2,10. Além disso, o controle deficiente da asma representa uma carga econômica maior para indivíduos, comunidade e sistemas de saúde, devido à utilização frequente dos serviços de emergência, a consultas não programadas, a hospitalizações e a medicamentos de custo elevado 9 . Ter asma não controlada, também, aumenta o risco de exacerbações que são responsáveis por maior morbidade da asma e precedem a maioria das mortes pela doença 1,11 .

Para compreender a importância da asma não controlada na morbimortalidade pela doença, diversos fatores são discutidos, tantos os gerais, como a poluição do ar e a sazonalidade climática, quanto os de caráter individual, como a presença de comorbidades, o uso inadequado dos inaladores, a falta de adesão ao tratamento, a superestimação do controle da doença e a educação deficiente dos pacientes e familiares, assim como as características genéticas 12,13,14,15,16,17. Dentre esses fatores de risco com maior evidência científica, merece destaque, neste estudo, a poluição do ar atmosférico.

A poluição do ar, interna ou externa, é responsável por inúmeros agravos respiratórios 18 . As investigações da ação da poluição do ar sobre a asma no Brasil têm levado em consideração grandes centros comerciais e industriais, voltados à poluição do ar em ambientes urbanos, diferindo do perfil de Primavera do Leste, Mato Grosso, local onde este estudo foi realizado. O município de médio porte tem sua economia voltada para o agronegócio e situa-se entre os dez que mais consomem agrotóxicos em suas lavouras, dentro de um estado que está em primeiro lugar na utilização desses produtos (Instituto Brasileiro de Geografia e Estatística. Sistema IBGE de Recuperação Automática: produção agrícola municipal. https://sidra.ibge.gov.br/home/ipp/brasil, acessado em 06/Nov/2019) 19. Diante disso, tem-se, como hipótese, que a exposição aos agrotóxicos na região pode aumentar a ocorrência de asma não controlada em escolares.

Nas principais culturas produzidas em Primavera do Leste (soja, milho e algodão), os princípios ativos mais utilizados, de 2012 a 2016, foram o glifosato, 2,4-D, metacloro, tebutiurom, trifluralina, paraquate, flutriafol, carbofurano, atrazina, clorpirifós, metomil, clomazona, diuron e etefon 19. Desses, a maioria são classificados como extremamente/altamente tóxicos à saúde humana. Tais substâncias químicas causam irritação da mucosa respiratória, promovem inflamação do epitélio brônquico, aumentam o número células inflamatórias, além de atuarem no sistema nervoso, interferindo na atividade de neurotransmissores, que reflete no funcionamento de órgãos efetores, no caso, pulmões 20,21. Com isso, ao potencializar o processo inflamatório das vias aéreas, essa forma de poluição do ar poderia perpetuar ou agravar a asma nos indivíduos expostos 22.

Assim, com o intuito de fornecer subsídios para elaboração de políticas públicas para melhorar o manejo da asma, este estudo tem o objetivo de analisar a influência da exposição aos agrotóxicos e outras condições sociodemográficas e relacionadas ao nascimento e à infância na ocorrência da asma não controlada em crianças e adolescentes em região de intensa atividade de agronegócio. 


\section{Métodos}

Trata-se de um estudo de caso controle, tendo, como ponto de partida, um inquérito populacional sobre prevalência e fatores de risco de asma e rinite em escolares 23 . O estudo de linha de base foi realizado com escolares pertencentes às faixas etárias de 6 a 7 anos e 13 a 14 anos, de 20 escolas do município, conforme o que preconiza o International Study of Asthma and Allergies in Childhood (ISAAC) 24. Após identificação dos potenciais asmáticos pela aplicação do questionário da Fase I do ISAAC a 3 mil alunos nas faixas etárias de 6 a 7 anos e 13 a 14 anos, foram aplicados outros dois questionários: da Fase II do ISAAC, para identificar os fatores de risco para asma, e questionário adicional, para avaliação de exposição aos agrotóxicos.

Foram considerados casos, escolares de 6 a 7 anos e de 13 a 14 anos classificados como de asma não controlada, seguindo os critérios do ISAAC para frequência de sintomas e maior gravidade da doença. Esses critérios foram utilizados em outras pesquisas e permaneceram inalterados e com o mesmo propósito nos estudos da Rede Global para Asma (GAN), reforçando a aplicabilidade deles $25,26,27,28,29$.

Os escolares que apresentaram resposta positiva a, pelo menos, uma das seguintes perguntas: "Nos últimos 12 (doze) meses, quantas crises de sibilos (chiados no peito) seu filho teve? Mais de três crises de sibilo no último ano" e/ou "Nos últimos 12 (doze) meses, com que frequência seu filho teve seu sono perturbado por chiado no peito? Mais de uma noite ou uma noite por semana de sono perturbado por sibilo" e/ou "Nos últimos 12 (doze) meses, o chiado do seu filho foi tão forte a ponto de impedir que ele conseguisse dizer mais de duas palavras entre cada respiração? Sim" - foram incluídos no grupo de casos como asma não controlada. Aqueles, classificados como de asma controlada foram, a partir de então, excluídos do banco de dados.

Para o grupo controle, foram considerados não asmáticos os escolares da mesma faixa etária que apresentaram resposta negativa à pergunta "Nos últimos 12 (doze) meses, seu filho teve sibilos (chiado no peito)?”. Após randomização dos escolares sem asma do estudo de base, estabeleceu-se relação de $1: 1$ entre casos e controles.

As variáveis independentes do estudo foram: sociodemográficas e econômicas, condições de nascimento e infância, antecedentes patológicos dos pais, exposição ambiental intradomiciliar e exposição ambiental aos agrotóxicos (Figura 1). As questões sobre exposição ambiental e ocupacional das crianças foram realizadas levando-se, em conta, o tempo de exposição (se atual ou anterior à pesquisa).

$\mathrm{Na}$ análise estatística, foram averiguadas as frequências absoluta e relativa das variáveis de interesse de estudo na análise descritiva, e, realizada a análise bivariada pelo método de qui-quadrado de Mantel-Haenszel para obtenção das odds ratio (OR) e seus respectivos intervalos de 95\% de confiança (IC95\%), para a identificação de associações brutas entre exposição e efeito.

Para a obtenção do modelo multivariado, utilizou-se o método de regressão logística, em que foram incluídas todas as variáveis que, nas análises brutas e estratificadas, apresentaram valor de $\mathrm{p}$ $<0,20$. Para inserção das variáveis, utilizou-se o modelo hierarquizado 30 (Figura 1), no qual, inicialmente, foram inseridas as variáveis consideradas distais, sendo mantidas, como ajuste, aquelas que apresentaram significância até o fim da análise. Na sequência, foram adicionadas as variáveis intermediárias, seguindo os mesmos critérios das distais, e, por fim, inseridas as proximais. A retirada das variáveis que não apresentaram significância do modelo de regressão deu-se por backward. No modelo final, permaneceram aquelas que apresentaram nível de significância menor ou igual 0,05. As análises estatísticas foram realizadas por meio do programa SPSS, versão 17.0 para Windows (https://www. ibm.com/).

A coleta de dados se deu por entrevistas realizadas em domicílios, no período de março a abril de 2016. Todos as entrevistas foram realizadas acompanhadas pelos pais ou responsáveis, após leitura e assinatura do Termo de Consentimento Livre e Esclarecido. Este estudo foi aprovado pelo Comitê de Ética em Pesquisa do Hospital Júlio Muller (CAAE: 3998.4114.0.0000.5541, parecer no 209 981471, de 11 de março de 2015), atendendo a Resolução no 196/1996 e a Resolução no 347/2005, do Conselho Nacional de Saúde. 
NÍVEL DISTAL

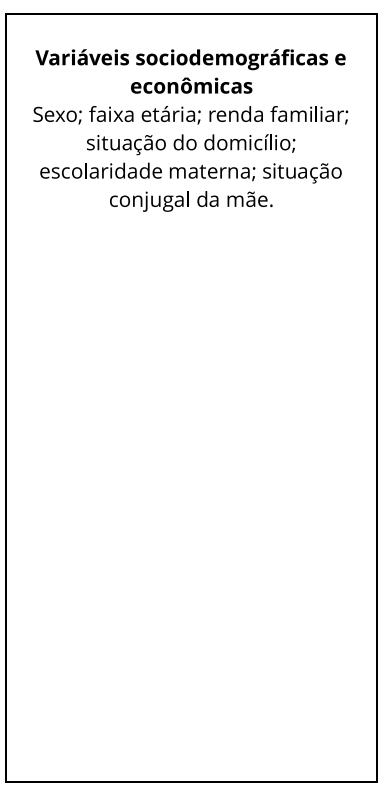

NÍVEL INTERMEDIÁRIO

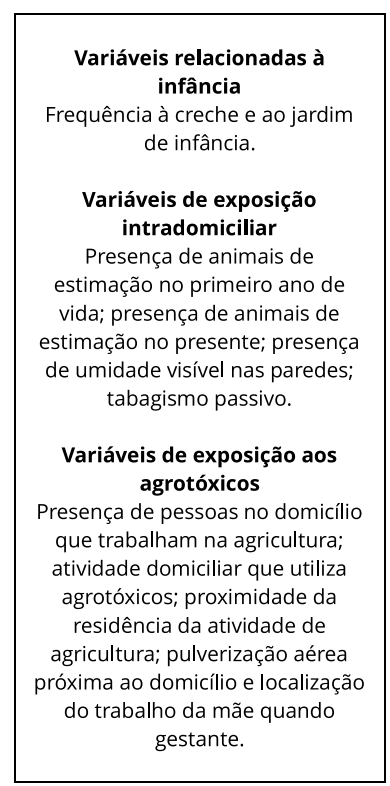

NÍVEL PROXIMAL

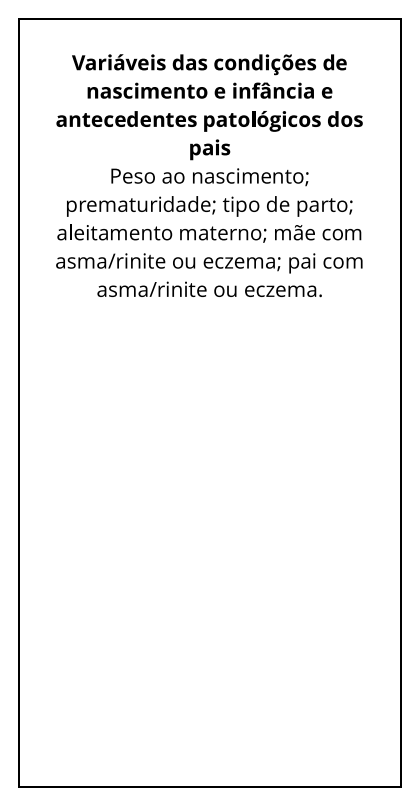

\section{Resultados}

O número de casos considerados como asma não controlada foi de 319 alunos, incluindo-se os controles na razão 1:1, totalizando 638 participantes no estudo. $\mathrm{Na}$ análise bivariada bruta, as variáveis sociodemográficas associadas à asma não controlada foram renda familiar maior que quatro salários mínimos (OR = 12,60; IC95\%: 8,61-18,43), escolaridade da mãe até Ensino Médio incompleto (OR = 10,33; IC95\%: 7,14-14,96) e situação conjugal materna sem companheiro dentro do grupo de casos $(\mathrm{OR}=2,54$; IC95\%: 1,76-3,67). As variáveis sexo e faixa etária não apresentaram associações estatisticamente significantes com asma não controlada (Tabela 1).

Ao avaliar as condições de nascimento e infância, prematuridade (OR = 3,2; IC95\%: 2,34-4,53), tipo de parto cesáreo ( $\mathrm{OR}=2,60$; IC95\%: 2,63-5,06), não aleitamento materno ( $\mathrm{OR}=8,3$; IC95\%: 5,73-12,16), frequentar creche (OR = 7,50; IC95\%: 5,26-10,85) e frequentar jardim de infância (OR = 2,30; IC95\%: 1,55-3,50) foram associados com asma não controlada. A variável peso ao nascer não apresentou diferença estatisticamente significante (Tabela 2).

Em relação aos antecedentes maternos, o grupo de casos se associou à presença de asma materna $(\mathrm{OR}=3,94$; IC95\%: 2,73-5,68) e à presença de eczema materno (OR = 2,22; IC95\%: 1,55-3,17), sem associação estatisticamente significante com presença de rinite alérgica. Quanto aos antecedentes paternos, a presença de asma não foi significante. Já as presenças de rinite $(\mathrm{OR}=0,07$; IC95\%: 0,040,11) e eczema $(\mathrm{OR}=0,30$; IC95\%: 0,14-0,65) foram inversamente associadas ao desfecho (Tabela 2).

A análise bivariada das variáveis de exposição intradomiciliar encontra-se na Tabela 3. A presença de animais no primeiro ano de vida, como cachorro ( $\mathrm{OR}=0,23$; IC95\%: 0,16-0,33) e gato $(\mathrm{OR}=0,62$; IC95\%: 0,45-0,85), assim como cachorro, no momento da pesquisa (OR = 0,10; IC95\%: 0,07-0,15), e gato, também no momento da pesquisa ( $\mathrm{OR}=0,62$; IC95\%: 0,45-0,85), foi associada inversamente com asma não controlada. O mesmo ocorreu com a presença de umidade visível na parede no presen- 
Tabela 1

Odds ratio (OR) e intervalo de 95\% de confiança (IC95\%) das variáveis sociodemográficas e econômicas associadas à asma não controlada entre crianças e adolescentes. Primavera do Leste, Mato Grosso, Brasil, 2016.

\begin{tabular}{|c|c|c|c|c|c|c|c|}
\hline \multirow[t]{2}{*}{ Variáveis } & \multicolumn{2}{|c|}{ Caso } & \multicolumn{2}{|c|}{ Controle } & \multirow[t]{2}{*}{ OR } & \multirow[t]{2}{*}{ IC95\% } & \multirow[t]{2}{*}{ Valor de $\mathrm{p}$} \\
\hline & $\mathbf{n}$ & $\%$ & $\mathbf{n}$ & $\%$ & & & \\
\hline \multicolumn{8}{|l|}{ Sexo } \\
\hline Masculino & 158 & 49,53 & 144 & 45,14 & 1,19 & $0,87-1,63$ & 0,27 \\
\hline Feminino & 161 & 50,47 & 175 & 54,86 & 1,00 & - & - \\
\hline \multicolumn{8}{|l|}{ Faixa etária (anos) } \\
\hline $6-7$ & 140 & 43,89 & 149 & 46,71 & 0,89 & $0,65-1,22$ & 0,47 \\
\hline $13-14$ & 179 & 56,11 & 170 & 53,29 & 1,00 & - & - \\
\hline \multicolumn{8}{|c|}{ Renda familiar (salários mínimos) } \\
\hline Mais de 4 & 264 & 82,76 & 88 & 27,59 & 12,6 & $8,61-18,43$ & $<0,01$ \\
\hline Até 4 & 55 & 17,24 & 231 & 72,41 & 1,00 & - & - \\
\hline \multicolumn{8}{|l|}{ Situação do domicílio } \\
\hline Rural & 31 & 9,72 & 35 & 10,97 & 0,87 & $0,52-1,46$ & 0,60 \\
\hline Urbano & 288 & 90,28 & 284 & 89,03 & 1,00 & - & - \\
\hline \multicolumn{8}{|l|}{ Escolaridade da mãe } \\
\hline Ensino Médio incompleto & 259 & 81,19 & 94 & 29,47 & 10,33 & $7,14-14,96$ & $<0,01$ \\
\hline Ensino Médio completo & 60 & 18,81 & 225 & 70,53 & 1,00 & - & - \\
\hline \multicolumn{8}{|l|}{ Situação conjugal da mãe } \\
\hline Sem companheiro & 112 & 35,11 & 56 & 17,55 & 2,54 & $1,76-3,67$ & $<0,01$ \\
\hline Com companheiro & 207 & 64,89 & 263 & 82,45 & 1,00 & - & - \\
\hline
\end{tabular}

te $(\mathrm{OR}=0,19$; IC95\%: 0,13-0,30). As variáveis relacionadas ao tabagismo não mostraram associação estatística neste estudo (dados não mostrados).

Observou-se que atividade domiciliar com utilização de agrotóxicos (OR = 1,71; IC95\%: 1,082,71), residir próximo ao local de atividades agrícolas ( $\mathrm{OR}=1,55$; IC95\%: 1,11-2,16), pulverização próxima ao domicílio (OR = 1,55; IC95\%: 1,11-2,16) e o fato de a mãe ter trabalhado em área rural durante a gestação $(\mathrm{OR}=1,69$; IC95\%: 1,07-2,67) foram associados aos casos (Tabela 3).

A análise do modelo final da regressão logística é mostrada na Tabela 4. As variáveis renda familiar maior que quatro salários mínimos, ter mãe com escolaridade até ensino médio incompleto e prematuridade mantiveram-se associadas ao grupo de casos. A variável ter baixo peso ao nascer, diferente da análise bivariada, mostrou-se associada na regressão logística (OR = 17,08; IC95\%: 5,52-52,90). Das variáveis relacionadas à exposição aos agrotóxicos, três mostraram-se associadas na regressão logística: presença de pessoas no domicílio que trabalham na agricultura (OR = 5,91; IC95\%: 2,11-16,53), proximidade do domicílio da atividade agrícola $(\mathrm{OR}=3,98$; IC95\%: 1,47-11,76) e pulverização aérea próxima ao domicílio (OR = 4,20; IC95\%: 1,49-11,87).

\section{Discussão}

Os resultados deste estudo demostraram a associação entre exposição aos agrotóxicos e asma não controlada em escolares, corroborando estudos internacionais sobre o tema 31,32,33,34. A proximidade do domicílio da atividade de agricultura, a pulverização aérea próxima às residências e a presença de trabalhadores da agricultura em casa permaneceram no modelo final de regressão logística, sendo essa última com maior efeito de associação.

Considerando que o município de estudo é todo circundado pelas lavouras, sendo provável que toda a população sofra os efeitos da aplicação dos agrotóxicos, a exposição domiciliar pela presença de pessoas que trabalham na agricultura torna-se importante por somar-se àquela mais generalizada. 
Tabela 2

Odds ratio (OR) e intervalo de 95\% de confiança (IC95\%) das condições de nascimento e infância e antecedentes patológicos dos pais associados à asma não controlada entre crianças e adolescentes. Primavera do Leste, Mato Grosso, Brasil, 2016.

\begin{tabular}{|c|c|c|c|c|c|c|c|}
\hline \multirow[t]{2}{*}{ Variáveis } & \multicolumn{2}{|c|}{ Caso } & \multicolumn{2}{|c|}{ Controle } & \multirow[t]{2}{*}{ OR } & \multirow[t]{2}{*}{ IC95\% } & \multirow[t]{2}{*}{ Valor de $\mathrm{p}$} \\
\hline & $\mathbf{n}$ & $\%$ & $\mathrm{n}$ & $\%$ & & & \\
\hline \multicolumn{8}{|c|}{ Peso ao nascimento (gramas) } \\
\hline Até 2.499 & 97 & 30,41 & 79 & 24,76 & 1,32 & $0,94-1,88$ & 0,11 \\
\hline$\geq 2.500$ & 222 & 69,59 & 240 & 75,24 & 1,00 & - & - \\
\hline \multicolumn{8}{|l|}{ Prematuridade } \\
\hline Pré-termo & 179 & 56,11 & 90 & 28,21 & 3,25 & $2,34-4,53$ & $<0,01$ \\
\hline Termo ou pós-termo & 140 & 43,89 & 229 & 71,79 & 1,00 & - & - \\
\hline \multicolumn{8}{|l|}{ Tipo de parto } \\
\hline Cesáreo & 196 & 61,44 & 97 & 30,41 & 3,65 & $2,63-5,06$ & $<0,01$ \\
\hline Normal & 123 & 38,56 & 222 & 69,59 & 1,00 & - & - \\
\hline \multicolumn{8}{|l|}{ Aleitamento materno } \\
\hline Não & 194 & 60,82 & 50 & 15,67 & 8,35 & $5,73-12,16$ & $<0,01$ \\
\hline Sim & 125 & 39,18 & 269 & 84,33 & 1,00 & - & - \\
\hline \multicolumn{8}{|l|}{ Frequência à creche } \\
\hline Sim & 259 & 81,19 & 116 & 36,36 & 7,55 & $5,26-10,85$ & $<0,01$ \\
\hline Não & 60 & 18,81 & 203 & 63,64 & 1,00 & - & - \\
\hline \multicolumn{8}{|c|}{ Frequência ao jardim de infância } \\
\hline Sim & 276 & 86,52 & 234 & 73,35 & 2,33 & $1,55-3,50$ & $<0,01$ \\
\hline Não & 43 & 13,48 & 85 & 26,65 & 1,00 & - & - \\
\hline \multicolumn{8}{|l|}{ Antecedentes familiares } \\
\hline \multicolumn{8}{|l|}{ Mãe com asma } \\
\hline Sim & 142 & 44,51 & 54 & 16,93 & 3,94 & $2,73-5,68$ & $<0,01$ \\
\hline Não & 177 & 55,49 & 265 & 83,07 & 1,00 & - & - \\
\hline \multicolumn{8}{|l|}{ Pai com asma } \\
\hline Sim & 68 & 21,32 & 64 & 20,06 & 1,08 & $0,74-1,58$ & 0,70 \\
\hline Não & 251 & 78,68 & 255 & 79,94 & 1,00 & - & - \\
\hline \multicolumn{8}{|l|}{ Mãe com rinite alérgica } \\
\hline Sim & 197 & 61,76 & 184 & 57,68 & 1,18 & $0,86-1,62$ & 0,29 \\
\hline Não & 122 & 38,24 & 135 & 42,32 & 1,00 & - & - \\
\hline \multicolumn{8}{|l|}{ Pai com rinite alérgica } \\
\hline Sim & 26 & 8,15 & 182 & 57,05 & 0,07 & $0,04-0,11$ & $<0,01$ \\
\hline Não & 293 & 91,85 & 137 & 42,95 & 1,00 & & \\
\hline \multicolumn{8}{|l|}{ Mãe com eczema } \\
\hline Sim & 114 & 35,74 & 64 & 20,06 & 2,22 & $1,55-3,17$ & $<0,01$ \\
\hline Não & 205 & 64,26 & 255 & 79,94 & 1,00 & - & - \\
\hline \multicolumn{8}{|l|}{ Pai com eczema } \\
\hline Sim & 9 & 2,82 & 28 & 8,78 & 0,30 & $0,14-0,65$ & $<0,01$ \\
\hline Não & 310 & 97,18 & 291 & 91,22 & 1,00 & - & - \\
\hline
\end{tabular}

A via de exposição paraocupacional, em que os agrotóxicos são transportados para o interior dos domicílios, por meio de roupas e acessórios dos trabalhadores rurais, contribui diretamente para a contaminação do ambiente interno ${ }^{35}$. Assim, os escolares expostos a essa via, possivelmente, recebem maior carga de poluentes, o que justifica o resultado encontrado.

A pulverização dos agrotóxicos, realizada principalmente por aviões e tratores nessa região, tem grande participação na contaminação do ambiente, que se dá pela deriva. Esse fenômeno refere-se ao 
Tabela 3

Odds ratio (OR) e intervalo de 95\% confiança (IC95\%) das variáveis de exposição intradomiciliar e exposição aos agrotóxicos associadas à asma não controlada entre crianças e adolescentes. Primavera do Leste, Mato Grosso, Brasil, 2016.

\begin{tabular}{|c|c|c|c|c|c|c|c|}
\hline \multirow[t]{2}{*}{ Variáveis } & \multicolumn{2}{|c|}{ Caso } & \multicolumn{2}{|c|}{ Controle } & \multirow[t]{2}{*}{ OR } & \multirow[t]{2}{*}{ IC95\% } & \multirow[t]{2}{*}{ Valor de $p$} \\
\hline & $\mathbf{n}$ & $\%$ & $\mathbf{n}$ & $\%$ & & & \\
\hline \multicolumn{8}{|c|}{ Presença de animais de estimação no primeiro ano de vida } \\
\hline \multicolumn{8}{|c|}{ Cachorro } \\
\hline Sim & 59 & 18,50 & 157 & 49,22 & 0,23 & $0,16-0,33$ & $<0,01$ \\
\hline Não & 260 & 81,50 & 162 & 50,78 & 1,00 & - & - \\
\hline \multicolumn{8}{|l|}{ Gato } \\
\hline Sim & 121 & 37,93 & 147 & 46,08 & 0,72 & $0,52-0,98$ & 0,04 \\
\hline Não & 198 & 62,07 & 172 & 53,92 & 1,00 & - & - \\
\hline \multicolumn{8}{|c|}{ Presença de animais de estimação no presente } \\
\hline \multicolumn{8}{|c|}{ Cachorro } \\
\hline Sim & 45 & 14,11 & 195 & 61,13 & 0,10 & $0,07-0,15$ & $<0,01$ \\
\hline Não & 274 & 85,89 & 124 & 38,87 & 1,00 & - & - \\
\hline \multicolumn{8}{|l|}{ Gato } \\
\hline Sim & 118 & 36,99 & 155 & 48,59 & 0,62 & $0,45-0,85$ & $<0,01$ \\
\hline Não & 201 & 63,01 & 164 & 51,41 & 1,00 & - & - \\
\hline \multicolumn{8}{|c|}{ Presença de umidade visível na parede no presente } \\
\hline Sim & 30 & 9,40 & 111 & 34,80 & 0,19 & $0,13-0,30$ & $<0,01$ \\
\hline Não & 289 & 90,60 & 208 & 65,20 & 1,00 & - & - \\
\hline \multicolumn{8}{|c|}{ Presença de pessoas no domicílio que trabalham na agricultura } \\
\hline Sim & 72 & 22,57 & 91 & 28,53 & 0,73 & $0,51-1,04$ & 0,08 \\
\hline Não & 247 & 77,43 & 228 & 71,47 & 1,00 & - & - \\
\hline \multicolumn{8}{|c|}{ Atividade domiciliar que utiliza agrotóxicos } \\
\hline Sim & 54 & 16,93 & 34 & 10,66 & 1,71 & $1,08-2,71$ & 0,02 \\
\hline Não & 265 & 83,07 & 285 & 89,34 & 1,00 & - & - \\
\hline \multicolumn{8}{|c|}{ Proximidade do domicílio da atividade de agricultura } \\
\hline Sim & 122 & 38,24 & 91 & 28,53 & 1,55 & $1,11-2,16$ & 0,01 \\
\hline Não & 197 & 61,76 & 228 & 71,47 & 1,00 & - & - \\
\hline \multicolumn{8}{|c|}{ Pulverização aérea próxima ao domicílio } \\
\hline Sim & 122 & 38,24 & 91 & 28,53 & 1,55 & $1,11-2,16$ & 0,01 \\
\hline Não & 197 & 61,76 & 228 & 71,47 & 1,00 & - & - \\
\hline \multicolumn{8}{|c|}{ Localização do trabalho da mãe quando estava gestante } \\
\hline Rural & 55 & 17,24 & 35 & 10,97 & 1,69 & $1,07-2,67$ & 0,02 \\
\hline Urbana & 264 & 82,76 & 284 & 89,03 & 1,00 & - & - \\
\hline
\end{tabular}

desvio de trajetória que impede as gotas do produto de atingirem seu alvo durante a aplicação ou à volatilização de resíduos de pesticidas, com consequente dispersão pelo vento, após sua aplicação 36 .

Estudo com análise de metarregressão evidenciou que as residências mais próximas a locais de pulverização de agrotóxicos e a presença de agricultores que haviam lidado com os químicos recentemente contribuíram para o aumento da concentração de agrotóxicos na poeira doméstica 37 . Somado a isso, existe o uso de agrotóxicos em domicílio para controle de pragas e jardinagem, que também esteve associado ao desfecho na análise bivariada. Outra pesquisa da exposição ocupacional, doméstica e residencial aos agrotóxicos demonstrou que as três diferentes exposições estiveram associadas à asma e a sintomas respiratórios em crianças 31 .

Nas principais culturas produzidas na região estudada (soja, milho e algodão), os princípios ativos mais utilizados, de 2012 a 2016, foram o glifosato, 2,4-D, metacloro, tebutiurom, trifluralina, 
Tabela 4

Modelo final da regressão logística. Primavera do Leste, Mato Grosso, Brasil, 2016.

\begin{tabular}{|c|c|c|c|}
\hline Variáveis & OR ajustada * & IC95\% & Valor de p \\
\hline Renda familiar (mais que 4 salários mínimos **/até 4 salários mínimos) & 14,36 & $8,89-23,20$ & $<0,01$ \\
\hline Escolaridade da mãe (Ensino Médio incompleto **/Ensino Médio completo) & 16,32 & $8,96-29,75$ & $<0,01$ \\
\hline Peso ao nascimento (Até $2.499 \mathrm{~g} * * / \geq 2.500 \mathrm{~g}$ ) & 17,08 & $5,52-52,90$ & $<0,01$ \\
\hline Prematuridade (pré-termo **/termo ou pós-termo) & 13,25 & $4,83-36,41$ & $<0,01$ \\
\hline Presença de pessoas no domicílio que trabalham na agricultura (sim **/não) & 5,91 & $2,11-16,53$ & $<0,0$ \\
\hline Proximidade do domicílio da atividade de agricultura (sim **/não) & 3,98 & $1,47-11,76$ & 0,01 \\
\hline Pulverização aérea próxima ao domicílio (sim **/não) & 4,20 & $1,49-11,87$ & 0,01 \\
\hline
\end{tabular}

IC95\%: intervalo de 95\% de confiança; OR: odds ratio.

* Ajustada por sexo, faixa etária dos estudantes e prematuridade;

** Categoria de referência.

paraquate, flutriafol, carbofurano, atrazina, clorpirifós, metomil, clomazona, diuron e etefon 19. Mais da metade desses agrotóxicos estiveram associados a sintomas respiratórios, como sibilância, em agricultores 38 . Asma atual e rinite foram relacionadas ao uso de 2,4-D, carbamatos e piretroides em agricultores em outra pesquisa 39 .

No estudo dos efeitos dos agrotóxicos organofosforados em crianças asmáticas moradoras de região rural com intensa atividade agrícola, foi encontrada relação entre a exposição aos químicos e a presença de marcadores inflamatórios presentes na asma em amostras de urina 40. Para os autores, os resultados evidenciam o papel dos pesticidas organofosforados como gatilhos ambientais agudos para o agravamento da morbidade da asma em crianças com a doença.

Diversos são os mecanismos pelos quais os agrotóxicos agridem as vias aéreas, e o principal deles é a inibição da enzima acetilcolinesterase, causada por organofosforados e carbamatos. Essa enzima é responsável pela degradação do neurotransmissor acetilcolina, seu bloqueio leva ao acúmulo do neurotransmissor nas terminações nervosas e ao estímulo aumentado no órgão efetor. No sistema respiratório, os receptores muscarínicos, quando estimulados, levam à constrição das vias aéreas. Nas exposições crônicas de baixo nível, para além dos mecanismos colinérgicos, os químicos organofosforados podem levar à hiper-responsividade das vias aéreas por interação direta com os receptores muscarínicos, o que resulta em broncoconstrição reflexa pela irritação pulmonar e secreção de muco pelas vias aéreas 21,40 .

Das variáveis sociodemográficas e econômicas estudadas e que se mantiveram associadas à asma não controlada no modelo final, chama atenção a renda familiar e a escolaridade materna. A associação de maior renda familiar com asma não controlada encontrada parece paradoxal quando se analisa a baixa escolaridade materna, que também se manteve associada. Estudos mostram que maior renda familiar acompanha o bom nível educacional da mãe, e isso é considerado como medida protetiva para qualquer tipo de agravo, pois, quanto melhor as condições maternas, melhor é o cuidado com a criança 41,42,43. Já a baixa escolaridade pode refletir no controle e na adesão ao tratamento da asma. As habilidades e conhecimentos alcançados por meio da educação podem afetar a receptividade às informações de saúde e a comunicação apropriada com os serviços de saúde e influenciar as decisões familiares nos cuidados à saúde e na prevenção de doenças 44 .

A prematuridade e o baixo peso ao nascer foram fortemente associados com a asma não controlada na regressão logística. Estudos confirmam esses resultados, sendo explicados pelo fato de as crianças com baixo peso terem menor maturação pulmonar, maior possibilidade de ter infecções respiratórias e de serem prematuras 45,46,47. As condições do nascimento podem interferir na formação do sistema respiratório causando comprometimento do crescimento pulmonar, vias aéreas menores, diminuição do volume pulmonar, aumento do risco de asma e outras doenças respiratórias 48 .

Nosso estudo possui algumas limitações, como o não pareamento individual dos casos e controles quanto a sexo e idade. Contudo, como se trata de grupos etários distintos de 6 a 7 anos e de 
13 a 14 anos, entende-se que as idades de ambos os grupos foram semelhantes. Além disso, o uso da randomização permitiu uma distribuição homogênea entre casos e controles quanto a essas duas variáveis. Outra limitação verificada com indivíduos expostos aos agrotóxicos é a impossibilidade de identificação de quais substâncias químicas foram responsáveis pelo desfecho, visto que a exposição é múltipla. Um fator limitante ainda é o do conceito de asma não controlada definido pela GINA (2019), que difere do instrumento utilizado (ISAAC), o qual não é específico para a coleta de dados dessa classificação da asma. Todavia, as questões abordadas no ISAAC oferecem medidas quantitativas alternativas da frequência dos sintomas e maior gravidade da asma, que podem indicar que a doença não está controlada, o que avaliza parcialmente nossa pesquisa.

Um ponto forte deste estudo é ser pioneiro na avaliação individual de exposição aos agrotóxicos e a ocorrência de asma não controlada em escolares em Mato Grosso, estado que, nos últimos anos, tem aumentado, de maneira exponencial, as atividades agropecuárias e o consumo de agrotóxicos, com consequente aumento da exposição dos trabalhadores e da população circunvizinha às grandes lavouras e aos químicos. Outro ponto a ser destacado refere-se ao uso do instrumento ISAAC, padronizado e validado internacionalmente, que é modelo para estudo da asma em todo o mundo.

\section{Conclusão}

Este estudo encontrou associação entre exposição de escolares aos agrotóxicos e asma não controlada. Também encontrou associações com as variáveis de ajuste maior renda familiar, ter mãe com menor escolaridade, prematuridade e baixo peso ao nascer com essa morbidade.

Esses resultados chamam a atenção para as políticas públicas a serem implantadas, tanto de saúde quanto ambientais, visando ao uso seguro, mesmo que pouco provável, de agrotóxicos no Brasil. Sugere-se que futuras investigações utilizando métodos de seguimento populacional e aferição direta de exposição sejam realizadas com intuito do subsídio de ações que diminuam o impacto e os efeitos da exposição de agrotóxicos na ocorrência de doenças respiratórias, em especial, a asma não controlada, em regiões de intensa atividade do agronegócio.

\section{Colaboradores}

C. B. Rocha contribuiu na concepção e desenho do estudo, análise e interpretação dos dados e redação das versões inicial e final do artigo. A. P. C. Nascimento colaborou na concepção, no desenho do estudo, na coleta de dados e aprovação da versão final do artigo. A. M. C. Silva participou da análise e interpretação dos dados, revisão crítica e aprovação da versão final do artigo. C. Botelho contribuiu na concepção e desenho do estudo, redação final do artigo, revisão crítica do manuscrito e aprovação da versão final.

\section{Informações adicionais}

ORCID: Cyndielle Barcelos da Rocha (0000-00022674-8469); Alessandra Pinheiro Costa Nascimento (0000-0001-8541-7240); Ageo Mário Cândido da Silva (0000-0001-5293-9413); Clóvis Botelho (0000-0002-2646-2639). 


\section{Referências}

1. Global Initiative for Asthma. Global strategy for asthma management and prevention. Updated 2019. https://ginasthma.org/wpcontent/uploads/2019/06/GINA-2019-mainreport-June-2019-wms.pdf (acessado em 11/ Nov/2019).

2. Neffen H, Fritscher C, Schacht FC, Levy G, Chiarella P, Soriano JB, et al. Asthma control in Latin America: the Asthma Insights and Reality in Latin America (AIRLA) survey. Rev Panam Salud Pública 2005; 17:191-7.

3. Demoly P, Annunziata K, Gubba E, Adamek L. Repeated cross-sectional survey of patient reported asthma control in Europe in the past 5 years. Eur Respir Rev 2012; 21:66-74.

4. Price D, Fletcher M, van der Molen T. Asthma control and management in 8,000 European patients: the REcognise Asthma and LInk to Symptoms and Experience (REALISE) survey. NPJ Prim Care Respir Med 2014; 24:14009.

5. Gebremariam TH, Binegdie AB, Mitiku AS, Ashagrie KGG, Huluka DK, Sherman CB, et al. Level of asthma control and risk factors for poor asthma control among clinic patients seen at a referral hospital in Addis Ababa, Ethiopia. BMC Res Notes 2017; 10:558.

6. Elnady HG, Sherif LS, Sabry RN, Zeid DA, Atta H, Hassanain AI. Relation of asthma control with quality of life among a sample of Egyptian asthmatic school children. Open Access Maced J Med Sci 2019; 7:2780-5.

7. Pizzichini MMM, Carvalho-Pinto RM, Cançado JED, Rubin AS, Cerci Neto A, Cardoso AP, et al. Recomendações para o manejo da asma da Sociedade Brasileira de Pneumologia e Tisiologia - 2020. J Bras Pneumol 2020; 46:e20190307.

8. Schmier JK, Manjunath R, Halpern MT, Jones ML, Thompson K, Diette GB. The impact of inadequately controlled asthma in urban children on quality of life and productivity. Ann Allergy Asthma Immunol 2007; 98:245-51.

9. Nunes C, Pereira AM, Morais-Almeida M. Asthma costs and social impact. Asthma Res Pract 2017; 3:1.

10. Ferrante G, La Grutta S. The burden of pediatric asthma. Front Pediatr 2018; 6:186.

11. Kirenga BJ, de Jong C, Mugenyi L, Katagira W, Muhofa A, Kamya MR, et al. Rates of asthma exacerbations and mortality and associated factors in Uganda: a 2-year prospective cohort study. Thorax 2018; 73:983-5.

12. Dick S, Doust E, Cowie H, Ayres JG, Turner S. Associations between environmental exposures and asthma control and exacerbations in young children: a systematic review. BMJ Open 2014; 4:e003827.

13. Nathan RA, Thompson PJ, Price D, Fabbri LM, Salvi S, González-Díaz S, et al. Taking aim at asthma around the world: global results of the asthma insight and management survey in the Asia-Pacific region, Latin America, Europe, Canada and the United States. J Allergy Clin Immunol Pract 2015; 3:734-42.
14. Braido F, Brusselle G, Guastalla D, Ingrassia E, Nicolini G, Price D. Determinants and impact of suboptimal asthma control in Europe: The International Cross-Sectional and Longitudinal Assessment On Asthma Control (LIAISON) study. Respir Res 2016; 17:51.

15. Xiang L, Zhao J, Zheng Y, Liu H, Hong J, Bao Y, et al. Uncontrolled asthma and its risk factors in Chinese children: a cross-sectional observational study. J Asthma 2016; 53:699-706.

16. Muñoz-Cano R, Torrego A, Bartra J, SanchézLópez J, Palomino R, Picado C, et al. Follow-up of patients with uncontrolled asthma: clinical features of asthma patients according to the level of control achieved (the COAS study). Eur Respir J 2017; 49:1501885.

17. Kabengele BO, Kayembe JN, Kayembe PK, Kashongue ZM, Kaba DK, Akilimali PZ. Factors associated with uncontrolled asthma in adult asthmatics in Kinshasa, Democratic Republic of Congo. PLoS One 2019; 14:e0215530.

18. Boechat L, Rios L. Poluição de ambientes internos. Rev Bras Alerg Imunopatol 2011; 34:83-9.

19. Pignati WA, Lima FANS, Lara SS, Correa MLM, Barbosa JR, Leão LHC, et al. Distribuição espacial do uso de agrotóxicos no Brasil: uma ferramenta para a vigilância em saúde. Ciênc Saúde Colet 2017; 22:3281-93.

20. Ye M, Beach J, Martin JW, Senthilselvan A. Occupational pesticide exposures and respiratory health. Int J Environ Res Public Health 2013; 10:6442-71.

21. Shaffo FC, Grodzki AC, Fryer AD, Lein PJ. Mechanisms of organophosphorus pesticide toxicity in the context of airway hyperreactivity and asthma. Am J Physiol Lung Cell Mol Physiol 2018; 315:L485-501.

22. Doust E, Ayres JG, Devereux G, Dick F, Crawford JO, Cowie $\mathrm{H}$, et al. Is pesticide exposure a cause of obstructive airways disease? Eur Respir Rev 2014; 23:180-92.

23. Nascimento APC. Prevalência e fatores de risco de asma e rinite em escolares de um município da mesorregião sudeste mato-grossense [Tese de Doutorado]. Cuiabá: Instituto de Saúde Coletiva, Universidade Federal de Mato Grosso; 2018.

24. Solé D, Wandalsen GF, Camelo-Nunes IC, Naspitz CK, Group I-B. Prevalence of symptoms of asthma, rhinitis, and atopic eczema among Brazilian children and adolescents identified by the International Study of Asthma and Allergies in Childhood (ISAAC) Phase 3. J Pediatr (Rio J.) 2006; 82:341-6.

25. Camelo-Nunes IC, Waldensen GF, Melo KC, Naspitz CK, Solé D. Prevalência de asma e de sintomas relacionados entre escolares de São Paulo, Brasil: 1996 a 1999. Rev Bras Alergia Imunopatol 2001; 24:77-89. 
26. Falade AG, Olawuyi JF, Osinusi K, Onadeko BO. Prevalence and severity of symptoms of asthma, allergic rhinoconjunctivitis, and atopic eczema in 6- to 7-year-old Nigerian primary school children: The International Study of Asthma and Allergies in Childhood. Med Princ Pract 2004; 13:20-5.

27. Kuschnir FC, Cunha AJLA, Braga DAC, Silveira HHN, Barroso MH, Aires ST. Asma em escolares de 13 e 14 anos do Município de Nova Iguaçu, Rio de Janeiro, Brasil: estimativas de prevalência, gravidade e diferenças de gênero. Cad Saúde Pública 2007; 23:919-26.

28. Lai CKW, Beasley R, Crane J, Foliaki S, Shah J, Weiland SK. Global variation in the prevalence and severity of asthma symptoms: Phase Three of the International Study of Asthma and Allergies in Childhood (ISAAC). Thorax 2009; 64:476-83.

29. Ellwood P, Asher MI, Billo NE, Bissell K, Chiang C-Y, Ellwood EM, et al. The Global Asthma Network rationale and methods for Phase I global surveillance: prevalence, severity, management and risk factors. Eur Respir J 2017; 49:1601605.

30. Lima S, Carvalho ML, Vasconcelos AGG. Proposta de modelo hierarquizado aplicado à investigação de fatores de risco de óbito infantil neonatal. Cad Saúde Pública 2008; 24:1910-6.

31. Salameh PR, Baldi I, Brochard P, Raherison C, Saleh BA, Salamon R. Respiratory symptoms in children and exposure to pesticides. Eur Resp J 2003; 22:507-12.

32. Duramad P, Harley K, Lipsett M, Bradman A, Eskenazi B, Holland NT, et al. Early environmental exposures and intracellular Th1/Th2 cytokine profiles in 24-month-old children living in an agricultural area. Environ Health Perspect 2006; 114:1916-22.

33. Raanan R, Harley KG, Balmes JR, Bradman A, Lipsett M, Eskenazi B. Early-life exposure to organophosphate pesticides and pediatric respiratory symptoms in the CHAMACOS cohort. Environ Health Perspect 2015; 123:17985.

34. Benka-Coker W, Loftus C, Karr C, Magzamen $\mathrm{S}$. Association of organophosphate pesticide exposure and a marker of asthma morbidity in an agricultural community. J Agromedicine 2020; 25:106-14.

35. Sacinelli PN. A exposição de crianças e adolescentes a agrotóxicos. In: Peres F, Moreira JC, organizadores. É veneno ou é remédio? Agrotóxicos, saúde e ambiente. Rio de Janeiro: Editora Fiocruz; 2003. p. 43-58.

36. Deziel NC, Friesen MC, Hoppin JA, Hines CJ, Thomas K, Freeman LE. A review of nonoccupational pathways for pesticide exposure in women living in agricultural areas. Environ Health Perspect 2015; 123:515-24.
37. Deziel NC, Freeman LE, Graubard BI, Jones RR, Hoppin JA, Thomas K, et al. Relative contributions of agricultural drift, para-occupational, and residential use exposure pathways to house dust pesticide concentrations: metaregression of published data. Environ Health Perspect 2017; 125:296-305.

38. Hoppin JA, Umbach DM, Long S, London SJ, Henneberger PK, Blair A, et al. Pescides are associated with allergic and non-allergic wheeze among male farmers. Environ Health Perspect 2017; 125:535-43.

39. Patel O, Syamlal G, Henneberger PK, Alarcon WA, Mazurek JM. Pesticide use, allergic rhinitis, and asthma among US farm operators. J Agromedicine 2018; 118:6072-8.

40. Banks CN, Lein PJ. A review of experimental evidence linking neurotoxic organophosphorus compounds and inflammation. Neurotoxicology 2012; 33:575-84.

41. Engle PL, Menon P, Haddad L. Care and nutrition: concepts and measurement. Washington DC: International Food Policy Research Institute; 1999.

42. Stephan AMS, Costa JSD. Conhecimento sobre asma das mães de crianças acometidas pela patologia, em área coberta pelo Programa Saúde da Família. Rev Bras Epidemiol 2009; 12:671-9.

43. Perosa GB, Amato IA, Rugolo LMSS, Ferrari GF, Oliveira MCFA. Qualidade de vida de crianças e adolescentes asmáticos: sua relação com estratégias de enfrentamento materno. Rev Paul Pediatr 2013; 31:145-51.

44. Galobardes B, Shaw M, Lawlor DA, Lynch JW, Smith GD. Indicators of socioeconomic position (part 1). J Epidemiol Community Health 2006; 60:7-12.

45. Chatkin MN, Menezes AMB. Associação entre baixo peso ao nascer e asma: uma revisão sistemática da literatura. Rev Panam Salud Pública 2005; 17:102-9.

46. Gonçalves ES, Mezzacappa-Filho F, Severino SD, Ribeiro MAGO, Marson FAL, Morcilo $\mathrm{AM}$, et al. Associação entre variáveis clínicas relacionadas à asma em escolares nascidos com muito baixo peso com e sem displasia broncopulmonar. Rev Paul Pediatr 2016; 34:271-80.

47. Den Dekker HT, Sonnenschein-van der Voort AM, Jongste JC, Anessi-Maesano I, Arshad $\mathrm{SH}, \mathrm{Barros} \mathrm{H}$, et al. Early growth characteristics and the risk of reduced lung function and asthma: a meta-analysis of 25,000 children. J Allergy Clin Immunol 2016; 137:1026-35.

48. Friedrich L, Corso AL, Jones MH. Prognóstico pulmonar em prematuros. J Pediatr (Rio J.) 2005; 81 Suppl 1:S79-88. 


\section{Abstract}

The study aimed to analyze factors associated with uncontrolled asthma in schoolchildren exposed to pesticides in a medium-sized municipality in the state of Mato Grosso, Brazil. This was a case-control study of children 6 to 7 and 13 to 14 years old in Primavera do Leste, in 2016. Cases were defined as schoolchildren that met the criteria for uncontrolled asthma based on International Study of Asthma and Allergies in Childhood (ISAAC) questions, and controls were selected from the same schools as the cases, after randomization, at a 1:1 ratio. Data collection used the questionnaires from Phases I and II of ISAAC and an additional questionnaire on pesticide exposure. Descriptive, bivariate, and logistic da e regression analyses were performed with the individual and environmental sociodemographic, and economic variables. 319 cases and 319 controls were selected, totaling 638 participants in the study. In the final da logistic model, the variables family income greater than 4 minimum wages $(O R=14.36 ; 95 \% C I$ : 8.89-23.20), maternal schooling up to incomplete secondary $(\mathrm{OR}=16.32$; 95\%CI: 8.96-29.75), prematurity $(O R=13.25 ; 95 \% C I: 4.83-36.41)$, and low birthweight (OR = 17.08; 95\%CI: 5.52-52.90) remained associated with uncontrolled asthma. Of the pesticide exposure variables, presence of household member working in agriculture $(O R=5.91$; 95\%CI: 2.11-16.53), living near farming activities $(\mathrm{OR}=3.98$; 95\%CI: 1.47-11.76), and spraying areas near the household $(\mathrm{OR}=4.20$; 95\% CI: 1.49-11.87) were related to the outcome. In this study, pesticides and sociodemographic, neonatal, and childhood conditions proved related to uncontrolled asthma in schoolchildren.

Asthma; Agrochemicals; Risk Factors

\section{Resumen}

El objetivo de este estudio es analizar los factores asociados al asma no controlado en escolares expuestos a pesticidas en un municipio de tamaño medio de Mato Grosso, Brazil. Se trata de un estudio de tipo caso-control, realizado con escolares de 6 a 7 años y de 13 a 14 de Primavera do Leste, en 2016. Se consideraron casos los escolares que cumplieron los criterios para asma no controlado, a través de preguntas del International Study of Asthma and Allergies in Childhood (ISAAC), los controles se seleccionaron a partir de las mismas escuelas que los casos, tras una aleatorización, en una relación de 1:1. Para la recogida de datos se utilizaron los cuestionarios de la Fase I y II del ISAAC, así como el cuestionario adicional para la exposición a los pesticidas. Se realizó un análisis descriptivo, bivariado y regresión logística de las variables sociodemográficas y económicas, así como individuales y ambientales. Se seleccionaron 319 casos y 319 controles, llegando a un total de 638 participantes en el estudio. En el modelo final de regresión logística las variables: renta familiar mayor que 4 salarios mínimos $(O R=14,36$; IC95\%: 8, 89-23,20), tener una madre con escolaridad hasta la enseñanza media incompleta $(O R=16,32$; IC95\%: 8,96-29,75), prematuridad $(\mathrm{OR}=13,25$; IC95\%: 4,83-36,41) $y$ bajo peso al nascer (OR = 17,08; IC95\%: 5,52$52,90)$ se mantuvieron asociadas al asma no controlado. Respecto a las variables de exposición a los pesticidas, la presencia de personas en el domicilio que trabajan en la agricultura $(O R=5,91$; IC95\%: 2,11-16,53), residir cerca de la actividad agrícola $(O R=3,98$; IC95\%: 1,47-11,76), así como la pulverización del aérea cercana al domicilio $(O R=4,20$; IC95\%: 1,49-11,87) se relacionaron con el resultado. En este estudio, los pesticidas y las condiciones sociodemográficas y de nacimiento e infancia se mostraron relacionadas con el asma no controlado en escolares.

Asma; Agrotóxicos; Factores de Riesgo
Recebido em 06/Abr/2020

Versão final representada em 28/Jul/2020

Aprovado em 23/Out/2020 